\title{
Free boundary and optimal stopping problems for American Asian options
}

\author{
Andrea Pascucci \\ Dipartimento di Matematica, Università di Bologna *
}

\begin{abstract}
We give a complete and self-contained proof of the existence of a strong solution to the free boundary and optimal stopping problems for pricing American path dependent options. The framework is sufficiently general to include geometric Asian options with non-constant volatility and recent path-dependent volatility models.
\end{abstract}

Keywords: American option, Asian option, free boundary problem, optimal stopping problem

Short running title: Free boundary and optimal stopping problems for American Asian options.

\section{Introduction}

In modern finance theory, the valuation of options with early exercise leads to optimal stopping problems which are equivalent to parabolic free boundary problems. Precisely, the price of an American option, with expiry date $T$ and payoff function $\varphi$, is the solution to the optimal stopping problem

$$
u(t, x)=\sup _{\tau \in \mathcal{T}_{t, T}} E\left[\varphi\left(X_{\tau}^{t, x}\right)\right],
$$

where $X$ is the diffusion, starting from $t, x$, that describes the dynamic of the underlying assets and $\mathcal{T}_{t, T}$ denotes the set of all stopping times with values in $[t, T]$. Equivalently, the price is determined by the solution to the free boundary problem

$$
\begin{cases}\max \{L u, \varphi-u\}=0, & \text { in }] 0, T\left[\times \mathbb{R}^{N},\right. \\ u(T, \cdot)=\varphi(T, \cdot), & \text { in } \mathbb{R}^{N},\end{cases}
$$

where $L$ is the Kolmogorov operator of $X$.

A rigorous theory of American options was first developed by Bensoussan [5], Karatzas [21], Jaillet, Lamberton and Lapeyre [19] by using the classical results by van Moerbeke [32, Bensoussan and Lions [6], Kinderlehrer and Stampacchia [23], Friedman [12] in the framework of parabolic PDEs. However, there are relevant kinds of American options, commonly traded in financial markets, whose modeling involves equations that are not uniformly parabolic and to

\footnotetext{
*Piazza di Porta S. Donato 5, 40126 Bologna (Italy), pascucci@dm.unibo.it
} 
which the classical theory does not apply. Two remarkable examples are given by average-rate (more commonly called Asian) options and recent path-dependent volatility models such as the stochastic volatility model by Hobson \& Rogers [15].

While there are several papers on the valuation of Asian options with early exercise (for instance, Barraquand and Pudet [2], Barles [1], Hansen and Jorgensen [14], Meyer [27], Wu, You and Kwok [33], Fu and Wu [13], Jiang and Dai[20], Ben-Ameur, Breton and L'Ecuyer [4], Marcozzi [26], Dai and Kwok [8], Huang and Thulasiram [17]), most of these are devoted to numerical issues (the development of numerical techniques for pricing and determining the exercise boundary) by some means assuming as established the existence and regularity of the solution to the free boundary or optimal stopping problem. To a certain extent, using the weak notion of viscosity solution, it is possible to obtain general existence results. Using the same techniques it is also possible to prove the uniform convergence of numerical schemes (cf. Barles [1] and Jiang and Dai [20]), though without having adequate control over the errors and the rate of convergence. As a matter of fact, if $L$ is a uniform parabolic operator, problem (1.2) is classically solved within the natural framework of the theory of Sobolev spaces and admits a strong solution. Indeed, it is well known that even in the Black-Scholes setting, a free boundary problem generally does not have a classical smooth solution and the regularity in some suitable Sobolev space is optimal.

In this paper we consider a quite general financial model, possibly correspondent to a degenerate PDE, that includes Asian options and path dependent volatility models as particular cases. We introduce a suitable functional setting and in this framework we prove the existence and uniqueness of a strong solution $u$ to the free boundary and optimal stopping problems. The regularity properties of $u$ are precisely stated in Section 4; roughly speaking, $u$ has weak second order derivatives in $L_{\text {loc }}^{p}$, for any $p \geq 1$, and locally Hölder continuous first order derivatives.

The outline for this paper is as follows. In Section 2, we briefly recall some known results for American Asian options in the Black-Scholes setting. In Section 3, we state the assumptions and examine some examples. Section 4 contains our main results regarding the existence of a strong solution to problem (1.2) and a Feynman-Kač type theorem connecting the free boundary and optimal stopping problems. In the Appendix, we review some basic facts about Kolmogorov PDEs associated with the linear SDEs and describe the functional setting suitable for our study.

\section{American Asian options in the Black-Scholes model}

Asian options are averaging options whose terminal payoff depends on some form of averaging prices of the underlying asset over a part or the whole life of the option. Let $r$ denote the constant interest rate and assume that the price of the underlying asset is modeled by a geometric Brownian motion

$$
d S_{t}=\mu S_{t} d t+\sigma S_{t} d W_{t}
$$

We denote by $M$ the path-dependent variable: for a arithmetic Asian option, we set

$$
M_{t}=\frac{A_{t}}{t}, \quad A_{t}=\int_{0}^{t} S_{s} d s
$$


and for a geometric Asian option,

$$
M_{t}=\exp \left(\frac{G_{t}}{t}\right), \quad G_{t}=\int_{0}^{t} \log \left(S_{s}\right) d s,
$$

Then the payoff function of a fixed-strike call Asian option is given by

$$
\varphi\left(t, S_{t}, M_{t}\right)=\left(M_{t}-K\right)^{+}
$$

where $K$ is the strike price; for a floating-strike call Asian option, the payoff is

$$
\varphi\left(t, S_{t}, M_{t}\right)=\left(S_{t}-M_{t}\right)^{+} .
$$

Arithmetic and geometric Asian options are commonly traded in specific markets (for instance, currency and commodity markets, cf. [14]) and were introduced to avoid the well-known problem related to European options that can be subject to price manipulations of the underlying asset near the maturity.

In case of arithmetic average (2.3), by usual no-arbitrage arguments we obtain the pricing PDE

$$
\frac{\sigma^{2} S^{2}}{2} \partial_{S S} u+r S \partial_{S} u+S \partial_{A} u+\partial_{t} u-r u=0,
$$

for the option price process $u\left(t, S_{t}, A_{t}\right)$. As usual, state augmentation converts the pathdependent problem for the Asian option into an equivalent path-independent and Markovian problem. However increasing the dimension generally leads to degenerate PDEs which are not uniformly parabolic: this is the case of equation (2.7) which only contains the second order partial derivatives with respect to one of the two "spatial variables".

On the other hand it is known that, in the particular Black-Scholes setting and for specific payoff functions, it possible to reduce the study of an Asian option to a PDE with only one state variable. More precisely, for the floating-strike Asian option, Ingersoll [18] proposes the change of variable $x=\frac{A}{S}$ : it is straightforward to show that $u=u(t, S, A)$ solves the Cauchy problem for (2.7) with final condition

$$
u\left(T, S_{T}, A_{T}\right)=\left(S_{T}-\frac{A_{T}}{T}\right)^{+}
$$

if and only of the function $U=U(t, x)$ defined by $u(t, S, A)=S U\left(t, \frac{A}{S}\right)$ solves the parabolic Cauchy problem in $\mathbb{R}^{2}$

$$
\begin{cases}\frac{\sigma^{2} x^{2}}{2} \partial_{x x} U+(1-r x) \partial_{x} U+\partial_{t} U=0, & t \in] 0, T[, x>0, \\ U(T, x)=\left(1-\frac{x}{T}\right)^{+}, & x>0 .\end{cases}
$$

A similar result holds for the corresponding free boundary problem in the early exercise case.

Analogously, for the fixed-strike Asian option, Rogers and Shi [30] implicitly propose the change of variable $x=\frac{A / T-K}{S}$ : in this case $u$ solves the Cauchy problem for (2.7) with final condition

$$
u\left(T, S_{T}, A_{T}\right)=\left(\frac{A_{T}}{T}-K\right)^{+},
$$


if and only of the function $U=U(t, x)$ defined by

$$
u(t, S, A)=S U\left(t, \frac{\frac{A}{T}-K}{S}\right)
$$

solves the degenerate Cauchy problem in $\mathbb{R}^{2}$

$$
\begin{cases}\frac{\sigma^{2} x^{2}}{2} \partial_{x x} u+\left(\frac{1}{T}-r x\right) \partial_{x} u+\partial_{t} u=0, & t \in] 0, T[, x \in \mathbb{R}, \\ u(T, x)=x^{+}, & x \in \mathbb{R} .\end{cases}
$$

Note that the PDE in (2.8) is not parabolic and degenerates at $x=0$.

We emphasize that reduction to a lower dimensional PDE is possible only under the rather restrictive hypotheses: namely, assuming that $S$ is a geometric Brownian motion and for the specific payoff functions in (2.5) and (2.6). More generally, reduction is possible if the payoff function has suitably homogeneity properties: for instance, $\varphi(t, S, M)=S \varphi(t, 1, M / S)$. The idea that degenerate diffusions can be reduced to lower-dimensional non-degenerate diffusions on a sub-manifold of the underlying asset space was carried on by Barraquand and Pudet [2].

In case of geometric Asian options, the pricing PDE for the value function $u=u(t, S, G)$ reads

$$
\frac{\sigma^{2} S^{2}}{2} \partial_{S S} u+r S \partial_{S} u+\log (S) \partial_{G} u+\partial_{t} u-r u=0, \quad t \in[0, T[, S, G>0 .
$$

By the change of variables (cf. [3])

$$
f(t, x, y)=e^{x \frac{2 r-\sigma^{2}}{2 \sqrt{2} \sigma}+t\left(\frac{2 r+\sigma^{2}}{2 \sqrt{2} \sigma}\right)^{2}} u\left(t, e^{\frac{\sigma x}{\sqrt{2}}}, \frac{\sigma y}{\sqrt{2}}\right)
$$

$u$ solves $(2.9)$ if and only if $f$ is a solution to

$$
\partial_{x x} f+x \partial_{y} f+\partial_{t} f=0, \quad t \in\left[0, T\left[,(x, y) \in \mathbb{R}^{2} .\right.\right.
$$

Even in this case, it seems that a reduction to a one-dimensional problem is not generally possible. On the other hand, in the next section we show that the process $\left(S_{t}, G_{t}\right)$ is non-degenerate and has an explicit, strictly positive transition density that is the fundamental solution of (2.9).

\section{Assumptions, preliminaries and examples}

We consider a quite general financial model where the dynamic of the $N$ state variables is driven by the stochastic differential equation

$$
d X_{t}=\left(B X_{t}+b\left(t, X_{t}\right)\right) d t+\sigma\left(t, X_{t}\right) d W_{t} .
$$

In (3.1) $W$ denotes a $d$-dimensional standard Wiener process, with $d \leq N$. To fix ideas, for an Asian option we have $N=2$ and $X$ is the two dimensional process whose components are the underlying price (in logarithmical scale) and the related average. We refer to Subsection 3.1 for further examples.

We assume the following structural condition: 
Assumption 3.1. $\sigma=\sigma(t, x)$ is a $N \times d$ matrix whose entries are bounded Hölder continuous functions. Moreover $B=\left(b_{i j}\right)$ is a $N \times N$ constant matrix and $b=\left(b_{1}, \ldots, b_{N}\right)$ is a bounded Hölder continuous vector valued function such that

$$
0=b_{d+1}=\cdots=b_{N} .
$$

By Remark 5.2 , the standard Hölder continuity hypothesis is equivalent to the more natural assumption $a_{i j}, b_{i} \in C_{B}^{\alpha}$ for some $\left.\alpha \in\right] 0,1$ [, where the Hölder space $C_{B}^{\alpha}$ is defined in Subsection 5.2. We also remark that our results straightforwardly generalize to the case $B=B(t)$ and $b_{d+1}(t), \ldots, b_{N}(t)$ measurable and bounded functions of time. Clearly, for $d=N$, condition (3.2) should be neglected.

Before stating the other main hypotheses, we recall some well-known facts about linear SDEs: for more details we refer, for instance, to [22], Chapter 5.6. Let $I_{d}$ denote the identity matrix in $\mathbb{R}^{d}$ and consider the $N \times d$ constant matrix defined, in block form, by

$$
\sigma_{0}=\left(\begin{array}{l}
I_{d} \\
0
\end{array}\right)
$$

Then, fixed $(y, s) \in \mathbb{R}^{N+1}$, the solution of the linear SDE

$$
d X_{t}^{s, y}=B X_{t}^{s, y} d t+\sigma_{0} d W_{t}, \quad X_{s}^{s, y}=y,
$$

is a Gaussian process with mean vector

$$
E\left[X_{t}^{s, y}\right]=e^{(t-s) B} y,
$$

and covariance matrix $\mathcal{C}_{0}(t-s)$ where

$$
\mathcal{C}_{0}(t)=\int_{0}^{t} e^{(t-\rho) B} \sigma_{0} \sigma_{0}^{*} e^{(t-\rho) B^{*}} d \rho, \quad t \geq 0 .
$$

Since $\sigma_{0}$ has dimension $N \times d$, the matrix $\mathcal{C}_{0}(t)$ is generally only positive semi-definite in $\mathbb{R}^{N}$, that is $X_{t}^{s, y}$ possibly has degenerate multi-normal distribution. The well-known Kalman condition from control theory provides an operative criterion for the positivity of $\mathcal{C}_{0}(t)$.

Theorem 3.2 (Kalman rank condition). The matrix $\mathcal{C}_{0}(t)$ is positive definite for $t>0$ if and only if

$$
\operatorname{rank}\left[\sigma_{0}, B \sigma_{0}, B^{2} \sigma_{0}, \ldots, B^{N-1} \sigma_{0}\right]=N .
$$

Incidentally the previous result shows that $\mathcal{C}_{0}(t)>0$ for some $t>0$ if and only if $\mathcal{C}_{0}(t)>0$ for every $t>0$. Then (3.5) ensures that $X^{s, y}$ has a Gaussian transition density

$$
G(s, y ; t, x)=\frac{1}{\sqrt{(2 \pi)^{N} \operatorname{det} \mathcal{C}_{0}(t-s)}} \exp \left(-\frac{1}{2}\left\langle\mathcal{C}_{0}^{-1}(t-s)\left(x-e^{(t-s) B} y\right), x-e^{(t-s) B} y\right) .\right.
$$

Furthermore $G$ is the fundamental solution of the Kolmogorov PDE associated to (3.4):

$$
K u(t, x):=\frac{1}{2} \sum_{i, j=1}^{d} \partial_{x_{i} x_{i}} u(t, x)+\sum_{i, j=1}^{N} b_{i j} x_{j} \partial_{x_{i}} u(t, x)+\partial_{t} u(t, x)=0,
$$


which, in compact form, reads

$$
K u=\frac{1}{2} \triangle_{\mathbb{R}^{d}} u+\sum_{i, j=1}^{N} b_{i j} x_{j} \partial_{x_{i}} u+\partial_{t} u=0 .
$$

We emphasize that generally (3.7) is not a uniformly parabolic PDE since $d \leq N$. In the Appendix we briefly review some basic results about Kolmogorov equations related to linear SDEs and verify that (3.5) is equivalent to the Hörmander condition [16], that is a non-degeneracy criterion, well-known in PDEs' theory.

Now we consider the general SDE (3.1) and state the second hypothesis:

Assumption 3.3. The matrix $\sigma \sigma^{*}$ takes the following block form

$$
\sigma \sigma^{*}=\left(\begin{array}{cc}
A & 0 \\
0 & 0
\end{array}\right)
$$

where $A=\left(a_{i j}\right)_{i, j=1, \ldots, d}$ is uniformly positive definite, i.e. there exists a positive constant $\Lambda$ such that

$$
\Lambda^{-1}|\eta|^{2}<\langle A(t, x) \eta, \eta\rangle<\Lambda|\eta|^{2}
$$

for any $\eta \in \mathbb{R}^{d}$ and $(t, x) \in \mathbb{R}^{N+1}$. Moreover the matrix $B$ satisfies the Kalman condition (3.5) with $\sigma_{0}$ as in (3.3).

Combining the results by Stroock \& Varadhan [31] and Di Francesco \& Pascucci [9], Assumptions 3.1 and 3.3 ensure the existence and uniqueness of a weak solution $\left(\Omega, \mathcal{F}, P,\left(\mathcal{F}_{s}\right), W, X\right)$ to SDE (3.1). Specifically, it is proved in [9] that the Kolmogorov operator of (3.1), that is

$$
L u(t, x)=\frac{1}{2} \sum_{i, j=1}^{d} a_{i j}(t, x) \partial_{x_{i} x_{j}} u(t, x)+\sum_{i, j=1}^{N} b_{i j} x_{j} \partial_{x_{i}} u(t, x)+\sum_{i=1}^{d} b_{i}(t, x) \partial_{x_{i}} u(t, x)+\partial_{t} u(t, x),
$$

has a fundamental solution $\Gamma=\Gamma(s, y ; t, x)$ which is the transition density of the weak solution of (3.1). Moreover the following Gaussian upper bound holds:

$$
\Gamma(s, y ; t, x) \leq C G_{0}(s, y ; t, x), \quad s<t, x, y \in \mathbb{R}^{N},
$$

where $G_{0}$ denotes a density of the form (3.6) and $C$ is a positive constant only dependent on $L$ and $t-s$. Precisely, $G_{0}$ is the transition density related to the linear SDE (3.4) with

$$
\sigma_{0}=\left(\begin{array}{c}
\Lambda I_{d} \\
0
\end{array}\right)
$$

and $\Lambda$ as in (3.9). For convenience, we rewrite operator $L$ in compact form:

$$
L=\frac{1}{2} \sum_{i, j=1}^{d} a_{i j} \partial_{x_{i} x_{j}}+\langle B x+b, \nabla\rangle+\partial_{t} .
$$

We consider the free boundary problem

$$
\begin{cases}\mathcal{L} u:=\max \{L u-r u-f, \varphi-u\}=0, & \text { in } \left.\mathcal{S}_{T}:=\right] 0, T\left[\times \mathbb{R}^{N},\right. \\ u(T, \cdot)=\varphi(T, \cdot), & \text { in } \mathbb{R}^{N} .\end{cases}
$$

We assume the following conditions on the coefficients. 
Assumption 3.4. The coefficients $r, f$ are bounded and Hölder continuous. The payoff function $\varphi$ is locally Lipschitz continuous on $\overline{\mathcal{S}_{T}}$ and for any compact subset $H$ of $\mathcal{S}_{T}$ there exists a constant $c \in \mathbb{R}$ such that

$$
\sum_{i, j=1}^{d} \eta_{i} \eta_{j} \partial_{x_{i} x_{j}} \varphi \geq c|\eta|^{2}, \quad \text { in } H, \eta \in \mathbb{R}^{d},
$$

in the distributional sense, that is

$$
\sum_{i, j=1}^{d} \eta_{i} \eta_{j} \int_{H} \partial_{x_{i} x_{j}} \psi(z) \varphi(z) d z \geq c|\eta|^{2} \int_{H} \psi(z) d z,
$$

for any $\eta \in \mathbb{R}^{d}$ and $\psi \in C_{0}^{\infty}(H), \psi \geq 0$.

The financial intuition underlying condition (3.13) is that the payoff $\varphi$ has to be a convex function (in a very weak sense) w.r.t. the first $d$ state variables corresponding, as we shall see in the examples, to the prices of underlying assets of the option.

We explicitly remark that any $C^{2}$ function satisfies condition (3.13) as well as any Lipschitz continuous function that is convex with respect to the first $d$ variables. In particular, the payoff functions of standard Call and Put Asian options are included. Note that $x \mapsto(x-K)^{+}$ satisfies (3.13) since its second order distributional derivative in $K$ is a Dirac delta which is "non-negative"; on the contrary the function $x \mapsto-(x-K)^{+}$does not satisfy (3.13) since its second order distributional derivative in $K$ is a minus Dirac delta which is not "bounded from below".

\subsection{Some examples}

Example 3.5 (Geometric Asian option with local volatility). In a local volatility model we assume that the logarithm $Z$ of the underlying asset has the following dynamic

$$
d Z_{t}=\mu\left(t, Z_{t}\right) d t+\sigma\left(t, Z_{t}\right) d W_{t}
$$

where $W$ is a standard one-dimensional Brownian motion. Then the average process $G$ in (2.4) for a geometric Asian option satisfies

$$
d G_{t}=Z_{t} d t .
$$

Adopting the notations (3.1)-(3.3), we have $1=d<N=2$ and

$$
X_{t}=\left(\begin{array}{c}
Z_{t} \\
G_{t}
\end{array}\right), \quad b=\left(\begin{array}{c}
\mu \\
0
\end{array}\right), \quad B=\left(\begin{array}{cc}
0 & 0 \\
1 & 0
\end{array}\right), \quad \sigma_{0}=\left(\begin{array}{c}
1 \\
0
\end{array}\right) .
$$

Moreover condition (3.9) is clearly satisfied with $A=\sigma^{2}$ whenever $\sigma$ is a (uniformly strictly) positive and bounded function. Finally, we have

$$
\left[\sigma_{0}, B \sigma_{0}\right]=\left(\begin{array}{cc}
1 & 0 \\
0 & 1
\end{array}\right),
$$

so that the Kalman rank condition and Assumption 3.3 are verified. We remark that our results can be generalized to accomodate the arithmetic average case as well: however this requires some non trivial (yet merely technical) question to be addressed. For this reason we treat separately this topic and plan to complete the study in a forthcoming paper. 
Example 3.6 (Geometric Asian option in the Heston stochastic volatility model). In the Heston stochastic volatility model we have $2=d<N=3$ and

$$
\begin{aligned}
d Z_{t} & =\left(\mu-\frac{\nu_{t}}{2}\right) d t+\sigma \sqrt{\nu_{t}} d W_{t}^{1}, \\
d \nu_{t} & =\left(a-\nu_{t}\right) d t+\eta \sqrt{\nu_{t}} d W_{t}^{2}, \\
d G_{t} & =Z_{t} d t
\end{aligned}
$$

where $\left(W^{1}, W^{2}\right)$ is a two-dimensional Brownian motion and $\mu, \sigma, a, \eta$ are positive parameters. In this case

$$
X_{t}=\left(\begin{array}{c}
Z_{t} \\
\nu_{t} \\
G_{t}
\end{array}\right), \quad b(t, z, \nu)=\left(\begin{array}{c}
\mu \\
a \\
0
\end{array}\right), \quad B=\left(\begin{array}{ccc}
0 & -\frac{1}{2} & 0 \\
0 & -1 & 0 \\
1 & 0 & 0
\end{array}\right), \quad \sigma_{0}=\left(\begin{array}{cc}
1 & 0 \\
0 & 1 \\
0 & 0
\end{array}\right),
$$

and again Assumption 3.3 is easily verified by the rank condition. We refer to the recent paper by Parrott and Clarke [28] for a numerical study of American Asian options under stochastic volatility.

Example 3.7 (Path dependent volatility). We consider an extension of the local volatility model in which the volatility is defined as a function of the whole trajectory of the underlying asset, not only in terms of the spot price. Path dependent volatility was first introduced by Hobson \& Rogers [15] and then generalized by Foschi and one of the authors [11: the main feature is that it generally leads to a complete market model. We refer to [11] for an empirical analysis which shows the effectiveness of the model and compares the hedging performance with respect to standard stochastic volatility models.

Let $\psi$ be an average weight that is a non-negative, piecewise continuous and integrable function on $]-\infty, T]$. We assume that $\psi$ is strictly positive in $[0, T]$ and we set

$$
\Psi(t)=\int_{-\infty}^{t} \psi(s) d s
$$

Then we define the average process as

$$
\left.\left.M_{t}=\frac{1}{\Psi(t)} \int_{-\infty}^{t} \psi(s) Z_{s} d s, \quad t \in\right] 0, T\right]
$$

where $Z_{t}=\log \left(e^{-r t} S_{t}\right)$ denotes the log-discounted price process. The Hobson \& Rogers model corresponds to the specification $\psi(t)=e^{\lambda t}$ for some positive parameter $\lambda$. Then by Itô formula we have

$$
d M_{t}=\frac{\varphi(t)}{\Phi(t)}\left(Z_{t}-M_{t}\right) d t
$$

Assuming the following dynamic for the log-price

$$
d Z_{t}=\mu\left(Z_{t}-M_{t}\right) d t+\sigma\left(Z_{t}-M_{t}\right) d W_{t},
$$

we obtain the pricing PDE

$$
\left.\frac{\sigma^{2}(z-m)}{2}\left(\partial_{z z} f-\partial_{z} f\right)+\frac{\varphi(t)}{\Phi(t)}(z-m) \partial_{m} f+\partial_{t} f=0, \quad(t, z, m) \in\right] 0, T\left[\times \mathbb{R}^{2} .\right.
$$


In this case $1=d<N=2$ and

$$
X_{t}=\left(\begin{array}{c}
Z_{t} \\
M_{t}
\end{array}\right), \quad b=\left(\begin{array}{c}
\mu \\
0
\end{array}\right), \quad B=\frac{\varphi(t)}{\Phi(t)}\left(\begin{array}{cc}
0 & 0 \\
1 & -1
\end{array}\right), \quad \sigma_{0}=\left(\begin{array}{c}
1 \\
0
\end{array}\right) .
$$

Assumptions 3.1 and 3.3 are readily verified.

\section{$4 \quad$ Free boundary and optimal stopping problems}

In this section we prove our main results regarding the free boundary problem (3.12) and the related optimal stopping problem for SDE (3.1). Throughout this section Assumptions 3.1, 3.3 and 3.4 are supposed to hold.

In order to introduce the notion of strong (super-)solution, we recall the definition of the Sobolev space $\mathcal{S}^{p}$ given in Subsection 5.2; for $p \geq 1, \mathcal{S}^{p}$ denotes the space of functions $u \in L^{p}$ such that $\partial_{x_{i}} u, \partial_{x_{i} x_{j}} u$ for $i=1, \ldots, d$ and

$$
Y u:=\langle B x, \nabla u\rangle+\partial_{t} u=\sum_{i, j=1}^{N} b_{i j} x_{j} \partial_{x_{i}} u+\partial_{t} u
$$

belong to $L^{p}$. As usual, given a domain $D$ in $\mathbb{R}^{N+1}, \mathcal{S}_{\text {loc }}^{p}(D)$ denotes the space of functions $u \in \mathcal{S}^{p}\left(D_{0}\right)$ for any compact subset $D_{0}$ of $D$. Let us also recall the following notation for a strip in $\mathbb{R}^{N+1}$ :

$$
\left.\mathcal{S}_{T}=\right] 0, T\left[\times \mathbb{R}^{N}\right. \text {. }
$$

Definition 4.1. A function $\left.\left.u \in \mathcal{S}_{\text {loc }}^{1}\left(\mathcal{S}_{T}\right) \cap C\left(\mathbb{R}^{N} \times\right] 0, T\right]\right)$ is a strong solution of problem (3.12) if $\mathcal{L} u=0$ almost everywhere in $\mathcal{S}_{T}$ and it attains the final datum pointwisely. A function $\left.\left.u \in \mathcal{S}_{\text {loc }}^{1}\left(\mathcal{S}_{T}\right) \cap C\left(\mathbb{R}^{N} \times\right] 0, T\right]\right)$ is a strong super-solution of problem $(3.12)$ if $\mathcal{L} u \leq 0$.

In Subsection 4.2 we prove the following existence result.

Theorem 4.2. If there exists a strong super-solution $\bar{u}$ of problem (3.12) then there also exists a strong solution $u$ of $(\overline{3.12})$ such that $u \leq \bar{u}$ in $\mathcal{S}_{T}$. Moreover $u \in \mathcal{S}_{\text {loc }}^{p}\left(\mathcal{S}_{T}\right)$ for any $p \geq 1$ and consequently, by the embedding theorem in Subsection [5.2, $u \in C_{B, \text { loc }}^{1, \alpha}\left(\mathcal{S}_{T}\right)$ for any $\left.\alpha \in\right] 0,1[$.

We remark that a supersolution to problem (3.12) exists in case of put options and more generally whenever $\varphi$ is a bounded function and $f \geq 0$. Indeed, in this case we can simply set $\bar{u}(x, t)=e^{-t\|r\|_{\infty}}\|\varphi\|_{\infty}$.

For unbounded payoffs, one can usually rely upon some financial consideration based on noarbitrage arguments: for instance, after the usual change of variable for the asset price $S=e^{x}$, a supersolution for an American call option, with payoff $\left(e^{x}-K\right)^{+}$, is simply given by $\bar{u}(t, x)=e^{x}$.

Regards to the regularity of the solution, recalling the definition of $C_{B}^{1, \alpha}$ in Subsection 5.2 , Theorem 4.2 shows that $u$ and its first $d$-derivatives $\partial_{x_{1}} u, \ldots, \partial_{x_{d}} u$ are Hölder continuous of exponent $\alpha$ for any $\alpha \in] 0,1[$. Since in [10] it is proved that strong solutions are also solutions in the viscosity sense, then Theorem 4.2 improves the known regularity results.

Now we denote by $X^{t, x}$ the solution to SDE (3.1) starting at time $t$ from $x \in \mathbb{R}^{N}$ and defined on the Wiener space $\left(\Omega, \mathcal{F}, P,\left(\mathcal{F}_{s}\right), W\right)$. We recall the following standard maximal estimate (cf., for instance, [22] or [29]):

$$
E\left[\exp \left(\lambda \sup _{t \leq s \leq T}\left|X_{s}^{t, x}\right|^{2}\right)\right]<\infty
$$


for a suitably small, positive constant $\lambda=\lambda(T, B, b, \Lambda, N)$ : more explicitly, it suffices

$$
\lambda \leq \frac{e^{-2 T\left(\|B\|+\|b\|_{\infty}\right)}}{2 T N \Lambda} .
$$

The following representation theorem for strong solutions holds.

Theorem 4.3. Let $u$ be a strong solution to the free boundary problem (3.12) such that

$$
|u(t, x)| \leq C e^{\lambda|x|^{2}}, \quad(t, x) \in \mathcal{S}_{T}
$$

form some constants $C, \lambda$ with $\lambda$ sufficiently small so that (4.2) holds. Then we have

$$
u(t, x)=\sup _{\tau \in \mathcal{T}_{t, T}} E\left[\varphi\left(\tau, X_{\tau}^{t, x}\right) e^{-\int_{t}^{\tau} r\left(s, X_{s}^{t, x}\right) d s}-\int_{t}^{\tau} f\left(s, X_{s}^{t, x}\right) e^{-\int_{t}^{s} r\left(\rho, X_{\rho}^{t, x}\right) d \rho} d s\right],
$$

where

$$
\mathcal{T}_{t, T}=\{\tau \in \mathcal{T} \mid \tau \in[t, T] \text { a.s. }\}
$$

and $\mathcal{T}$ is the set of all stopping times with respect to the filtration $\left(\mathcal{F}_{s}\right)$. In particular such a solution is unique.

In the next subsections we prove Theorem 4.3 and present a detailed outline of the proof of Theorem 4.2: for a complete study of the related obstacle problem, we refer to the recent paper [10] in collaboration with Di Francesco and Polidoro.

\subsection{Proof of Theorem 4.3}

For simplicity, we only consider the case $r=f=0$. As in the classical case the proof is based on Itô formula. However we remark that a strong solution $u$ does not have the required regularity in order to apply the Itô formula directly. Then in order to exploit the weak interior regularity properties of $u$, we employ a truncation and regularization technique.

We set $B_{R}=\left\{x \in \mathbb{R}^{N}|| x \mid<R\right\}, R>0$, and for $x \in B_{R}$ denote by $\tau_{R}$ the first exit time of $X^{t, x}$ from $B_{R}$. It is well-known that, since $\sigma$ is not totally degenerate, $E\left[\tau_{R}\right]$ is finite.

As a first step we prove the following result: for every $(t, x) \in] 0, T\left[\times B_{R}\right.$ and $\tau \in \mathcal{T}$ such that $t \leq \tau \leq \tau_{R}$ a.s., it holds

$$
u(t, x)=E\left[u\left(\tau, X_{\tau}^{t, x}\right)-\int_{t}^{\tau} L u\left(s, X_{s}^{t, x}\right) d s\right] .
$$

Indeed fixed $\varepsilon$, positive and suitably small, we consider a function $u^{\varepsilon, R}$ on $\mathbb{R}^{N+1}$ with compact support and such that $u^{\varepsilon, R}=u$ on $] t, T-\varepsilon\left[\times B_{R}\right.$. Moreover we denote by $\left(u^{\varepsilon, R, n}\right)_{n \in \mathbb{N}}$ a regularizing sequence obtained by convolution of $u^{\varepsilon, R}$ with the usual mollifiers: then, for any $p \geq 1$, $u^{\varepsilon, R, n} \in \mathcal{S}^{p}\left(\mathbb{R}^{N+1}\right)$ and

$$
\lim _{n \rightarrow \infty}\left\|L u^{\varepsilon, R, n}-L u^{\varepsilon, R}\right\|_{L^{p}(] t, T-\varepsilon\left[\times B_{R}\right)}=0 .
$$

By the standard Itô formula applied to the smooth function $u^{\varepsilon, R, n}$ we have

$$
u^{\varepsilon, R, n}\left(\tau, X_{\tau}^{t, x}\right)=u^{\varepsilon, R, n}(t, x)+\int_{t}^{\tau} L u^{\varepsilon, R, n}\left(s, X_{s}^{t, x}\right) d s+\int_{t}^{\tau} \nabla u^{\varepsilon, R, n}\left(s, X_{s}^{t, x}\right) \sigma\left(s, X_{s}^{t, x}\right) d W_{s}
$$


for $\tau \in \mathcal{T}$ such that $t \leq \tau \leq \tau_{R} \wedge(T-\varepsilon)$ a.s. Since $\left(\nabla u^{\varepsilon, R, n}\right) \sigma$ is a bounded function on ]$t, T-\varepsilon\left[\times B_{R}\right.$, we have

$$
E\left[\int_{t}^{\tau} \nabla u^{\varepsilon, R, n}\left(s, X_{s}^{t, x}\right) \sigma\left(s, X_{s}^{t, x}\right) d W_{s}\right]=0
$$

Moreover we have

$$
\lim _{n \rightarrow \infty} u^{\varepsilon, R, n}(t, x)=u^{\varepsilon, R}(t, x),
$$

and, by dominated convergence,

$$
\lim _{n \rightarrow \infty} E\left[u^{\varepsilon, R, n}\left(\tau, X_{\tau}^{t, x}\right)\right]=E\left[u^{\varepsilon, R}\left(\tau, X_{\tau}^{t, x}\right)\right] .
$$

Next we prove the convergence of the deterministic integral in (4.7). First we remark that, by the Gaussian estimate (3.10), the transition density of $X^{t, x}$ satisfies:

$$
\Gamma(t, x ; \cdot, \cdot) \in L^{\bar{q}}(] t, T\left[\times B_{R}\right),
$$

for some $\bar{q}>1$. We show (4.8) at the end of this subsection and, taking it for granted, first conclude the proof of the theorem. We have

$$
\begin{aligned}
\mid E\left[\int_{t}^{\tau} L u^{\varepsilon, R, n}\left(s, X_{s}^{t, x}\right) d s\right] & -E\left[\int_{t}^{\tau} L u^{\varepsilon, R}\left(s, X_{s}^{t, x}\right) d s\right] \mid \\
& \leq E\left[\int_{t}^{\tau}\left|L u^{\varepsilon, R, n}\left(s, X_{s}^{t, x}\right)-L u^{\varepsilon, R}\left(s, X_{s}^{t, x}\right)\right| d s\right] \leq
\end{aligned}
$$

(since $\tau \leq \tau_{R}$ )

$$
\begin{aligned}
& \leq E\left[\int_{t}^{T-\varepsilon}\left|L u^{\varepsilon, R, n}\left(s, X_{s}^{t, x}\right)-L u^{\varepsilon, R}\left(s, X_{s}^{t, x}\right)\right| \mathbb{1}_{\left\{\left|X_{s}^{t, x}\right| \leq R\right\}} d s\right] \\
& =\int_{t}^{T-\varepsilon} \int_{B_{R}}\left|L u^{\varepsilon, R, n}(s, y)-L u^{\varepsilon, R}(s, y)\right| \Gamma(t, x ; s, y) d y d s \leq
\end{aligned}
$$

(by Hölder inequality, denoting by $\bar{p}$ the conjugate exponent of $\bar{q}$ in (4.8))

$$
\leq\left\|L u^{\varepsilon, R, n}-L u^{\varepsilon, R}\right\|_{L^{\bar{p}}(] t, T-\varepsilon\left[\times B_{R}\right)}\|\Gamma(t, x ; \cdot, \cdot)\|_{L^{\bar{q}}(] t, T-\varepsilon\left[\times B_{R}\right)},
$$

and, by (4.6) and (4.8), we obtain

$$
\lim _{n \rightarrow \infty} E\left[\int_{t}^{\tau} L u^{\varepsilon, R, n}\left(s, X_{s}^{t, x}\right) d s\right]=E\left[\int_{t}^{\tau} L u^{\varepsilon, R}\left(s, X_{s}^{t, x}\right) d s\right] .
$$

This concludes the proof of (4.5), since $u^{\varepsilon, R}=u$ on $] t, T-\varepsilon\left[\times B_{R}\right.$ and $\varepsilon>0$ is arbitrary.

Next, since $L u \leq 0$ a.e. and the law of $X^{t, x}$ is absolutely continuous with respect to the Lebesgue measure, we have

$$
E\left[\int_{t}^{\tau} L u\left(s, X_{s}^{t, x}\right) d s\right] \leq 0
$$


for any $\tau \in \mathcal{T}_{t, T}$, and from (4.5) we infer

$$
u(t, x) \geq E\left[u\left(\tau \wedge \tau_{R}, X_{\tau \wedge \tau_{R}}^{t, x}\right)\right],
$$

for any $\tau \in \mathcal{T}_{t, T}$. Next we pass to the limit as $R \rightarrow+\infty$ : we have

$$
\lim _{R \rightarrow+\infty} \tau \wedge \tau_{R}=\tau
$$

pointwisely and, by the growth assumption (4.3),

$$
\left|u\left(\tau \wedge \tau_{R}, X_{\tau \wedge \tau_{R}}^{t, x}\right)\right| \leq C \exp \left(\lambda \sup _{t \leq s \leq T}\left|X_{s}^{t, x}\right|^{2}\right) .
$$

By (4.2) the exponential in the right hand side of the previous estimate is integrable: thus Lebesgue theorem applies and we deduce from (4.9), as $R \rightarrow+\infty$,

$$
u(t, x) \geq E\left[u\left(\tau, X_{\tau}^{t, x}\right)\right] \geq E\left[\varphi\left(\tau, X_{\tau}^{t, x}\right)\right] .
$$

This shows that

$$
u(t, x) \geq \sup _{\tau \in \mathcal{T}_{t, T}} E\left[\varphi\left(\tau, X_{\tau}^{t, x}\right)\right] .
$$

We conclude the proof by putting

$$
\tau_{0}=\inf \left\{s \in[t, T] \mid u\left(s, X_{s}^{t, x}\right)=\varphi\left(s, X_{s}^{t, x}\right)\right\} .
$$

Since $L u=0$ a.e. where $u>\varphi$, it holds

$$
E\left[\int_{t}^{\tau_{0} \wedge \tau_{R}} L u\left(s, X_{s}^{t, x}\right) d s\right]=0,
$$

and from (4.5) we infer

$$
u(t, x)=E\left[u\left(\tau_{0} \wedge \tau_{R}, X_{\tau_{0} \wedge \tau_{R}}^{t, x}\right)\right] .
$$

Repeating the previous argument to pass to the limit in $R$, we obtain

$$
u(t, x)=E\left[u\left(\tau_{0}, X_{\tau_{0}}^{t, x}\right)\right]=E\left[\varphi\left(\tau_{0}, X_{\tau_{0}}^{t, x}\right)\right] .
$$

In order to conclude the proof of Theorem 4.3, it remains to show (4.8). By estimate (3.10), it suffices to prove that $G(t, x ; \cdot, \cdot)$ in (3.6) belongs to $L^{q}(] t, T\left[\times \mathbb{R}^{N}\right)$ for $q<1+\frac{2}{Q}$, where $Q$ is the homogeneous dimension of $\mathbb{R}^{N}$ defined in (5.7). We have, for a suitable constant $c$,

$$
\begin{aligned}
& \int_{t}^{T} \int_{\mathbb{R}^{N}} G(t, x ; s, y)^{q} d y d s \\
& =\int_{t}^{T} \int_{\mathbb{R}^{N}} \frac{c}{\left(\operatorname{det} \mathcal{C}_{0}(s-t)\right)^{\frac{q}{2}}} \exp \left(-\frac{q}{2}\left\langle\mathcal{C}_{0}^{-1}(s-t)\left(y-e^{(s-t) B} x\right), y-e^{(s-t) B} x\right) d y d s=\right.
\end{aligned}
$$

(by the change of variables $\eta=\mathcal{C}_{0}^{-\frac{1}{2}}(s-t)\left(y-e^{(s-t) B} x\right)$ )

$$
=\int_{t}^{T} \frac{c}{\left(\operatorname{det} \mathcal{C}_{0}(s-t)\right)^{\frac{q-1}{2}}} d s \int_{\mathbb{R}^{N}} e^{-\frac{|\eta|^{2}}{2}} d \eta
$$

Then the thesis follows from the fact (see, for instance, Sect.2 in [9]) that

$$
\operatorname{det} \mathcal{C}_{0}(s-t)=O\left((s-t)^{Q}\right), \quad \text { as } s \rightarrow t .
$$




\subsection{Free boundary problem}

A solution of problem (3.12) can be obtained as the limit of strong solutions to a sequence of free boundary problems on bounded cylinders of the form $] 0, T\left[\times H_{n}\right.$, where $\left(H_{n}\right)$ is an increasing covering of $\mathbb{R}^{N}$. The proof of this simple and quite general fact can be found, for instance, in [10], Theorem 4.1. Thus here we only examine the free boundary problem on a bounded cylinder. Precisely, we prove the existence of a strong solution to problem

$$
\left\{\begin{array}{l}
\max \{L u-r u-f, \varphi-u\}=0, \quad \text { in } H(T):=] 0, T[\times H, \\
\left.u\right|_{\partial_{P} H(T)}=\varphi,
\end{array}\right.
$$

where $H$ is a bounded domain of $\mathbb{R}^{N}$ and

$$
\partial_{P} H(T):=\partial H(T) \backslash(\{T\} \times H)
$$

is the parabolic boundary of $H(T)$. Hereafter we assume that $H(T)$ is a regular domain in the sense that the standard initial-boundary problem for $L$ on $H(T)$ has a solution: a well-known sufficient condition for this, is the existence of a so-called barrier function at any point of the parabolic boundary. We recall that a barrier $w$ at $\zeta \in \partial_{P} H(T)$, is a smooth function defined in $V \cap \overline{H(T)}$ where $V$ is a suitable neighborhood of $\zeta$, such that $L w \leq-1$ in $V \cap H(T), w>0$ in $V \cap \overline{H(T)} \backslash\{\zeta\}$ and $w(\zeta)=0$.

Theorem 4.4. Problem (4.11) has a strong solution $u \in \mathcal{S}_{\text {loc }}^{1}(H(T)) \cap C(\overline{H(T)})$. Moreover $u \in \mathcal{S}_{\text {loc }}^{p}(H(T))$ for any $p>1$.

Proof. The proof is based on a penalization technique. We consider a family $\left(\beta_{\varepsilon}\right)_{\varepsilon \in] 0,1[}$ of smooth functions such that, for any $\varepsilon$, the function $\beta_{\varepsilon}$ is increasing, bounded on $\mathbb{R}$ and has bounded first order derivative. Moreover $\beta_{\varepsilon}(0)=0$,

$$
\beta_{\varepsilon}(s) \leq \varepsilon, \quad s>0, \quad \text { and } \quad \lim _{\varepsilon \rightarrow 0} \beta_{\varepsilon}(s)=-\infty, \quad s<0 .
$$

We also denote by $L^{\delta}, \delta>0$, the operator obtained by regularizing the coefficients of $L$. Besides $\varphi^{\delta}, r^{\delta}$ and $f^{\delta}$ respectively denote the regularizations of $\varphi, r$ and $f$.

By a general result for quasilinear equations (see, for instance, Theorem 3.2 in [10]), there exists a classical solution $\left.u_{\varepsilon, \delta} \in C_{B}^{2, \alpha}(H(T)) \cap C(\overline{H(T)}), \alpha \in\right] 0,1$, to the penalized and regularized problem

$$
\left\{\begin{array}{l}
L^{\delta} u-r^{\delta} u=f^{\delta}+\beta_{\varepsilon}\left(u-\varphi^{\delta}\right), \quad \text { in } H(T), \\
\left.u\right|_{\partial_{P} H(T)}=\varphi^{\delta}
\end{array}\right.
$$

The crucial step consists in proving the uniform boundedness of the penalization term:

$$
\left|\beta_{\varepsilon}\left(u_{\varepsilon, \delta}-\varphi^{\delta}\right)\right| \leq c, \quad \text { in } H(T),
$$

with $c$ independent of $\varepsilon$ and $\delta$.

Since by construction $\beta_{\varepsilon} \leq \varepsilon$, it suffices to prove the lower bound in (4.12). By continuity, $\beta_{\varepsilon}\left(u_{\varepsilon, \delta}-\varphi^{\delta}\right)$ has a minimum $\zeta$ in $\overline{H(T)}$ and we may suppose

$$
\beta_{\varepsilon}\left(u_{\varepsilon, \delta}(\zeta)-\varphi^{\delta}(\zeta)\right) \leq 0,
$$


otherwise there is nothing to prove. Now, if $\zeta \in \partial_{P} H(T)$ then

$$
\beta_{\varepsilon}\left(u_{\varepsilon, \delta}(\zeta)-\varphi^{\delta}(\zeta)\right)=\beta_{\varepsilon}(0)=0 .
$$

On the other hand, if $\zeta \in H(T)$, then we recall that $\beta_{\varepsilon}$ is increasing and consequently $u_{\varepsilon, \delta}-\varphi^{\delta}$ also has a (negative) minimum in $\zeta$. Thus, since it is not restrictive to assume $r \geq 0$, we have

$$
L^{\delta} u_{\varepsilon, \delta}(\zeta)-L^{\delta} \varphi^{\delta}(\zeta) \geq 0 \geq r(\zeta)\left(u_{\varepsilon, \delta}(\zeta)-\varphi^{\delta}(\zeta)\right)
$$

Now by the weak convexity condition (3.13) on $\varphi$ in Assumption 3.4, we have that $L^{\delta} \varphi^{\delta}(\zeta)$ is bounded uniformly in $\delta$. Therefore, by (4.13), we deduce

$$
\begin{aligned}
\beta_{\varepsilon}\left(u_{\varepsilon, \delta}(\zeta)-\varphi^{\delta}(\zeta)\right) & =L^{\delta} u_{\varepsilon, \delta}(\zeta)-r^{\delta}(\zeta) u_{\varepsilon, \delta}(\zeta)-f^{\delta}(\zeta) \\
& \geq L^{\delta} \varphi^{\delta}(\zeta)-r^{\delta}(\zeta) \varphi^{\delta}(\zeta)-f^{\delta}(\zeta) \geq c,
\end{aligned}
$$

where $c$ is a constant independent on $\varepsilon, \delta$ and this proves (4.12).

Next, we use the $\mathcal{S}^{p}$ interior estimate (5.8) combined with (4.12), to infer that, for every $D \subset \subset H(T)$ and $p \geq 1$, the norm $\left\|u_{\varepsilon, \delta}\right\|_{\mathcal{S}^{p}(D)}$ is bounded uniformly in $\varepsilon$ and $\delta$. It follows that $\left(u_{\varepsilon, \delta}\right)$ converges as $\varepsilon, \delta \rightarrow 0$, weakly in $\mathcal{S}^{p}$ (and in $C_{B}^{1, \alpha}$, by (5.9)) on compact subsets of $H(T)$ to a function $u$. Moreover

$$
\limsup _{\varepsilon, \delta \rightarrow 0} \beta_{\varepsilon}\left(u_{\varepsilon, \delta}-\varphi^{\delta}\right) \leq 0,
$$

so that $L u-r u \leq f$ a.e. in $H(T)$. On the other hand, $L u-r u=f$ a.e. in the set $\{u>\varphi\}$. Finally, it is straightforward to verify that $u \in C(\overline{H(T)})$ and assumes the initial-boundary conditions, by using standard arguments based on the maximum principle and barrier functions.

\section{Appendix}

We review some basic facts about the Kolmogorov operator in (3.7) associated with the linear SDE (3.4). With compact notations, the operator takes the form

$$
K=\frac{1}{2} \triangle_{\mathbb{R}^{d}}+Y
$$

where $1 \leq d \leq N$ and $Y$ is as in (4.1). In the sequel we assume that the Kalman rank condition (3.5) holds. We also systematically write $z=(t, x)$ and $\zeta=(s, y)$ to denote points in $\mathbb{R}^{N+1}$.

The main purpose of this section is to describe the non-Euclidean group and metric structures, induced by $K$ on $\mathbb{R}^{N+1}$, which provide the natural framework for the study of the regularity properties of the operator. This structure was first studied by Lanconelli and Polidoro [24]. Secondly, we define some non-standard Sobolev and Hölder space adapted to this non-Euclidean setting and state the basic a-priori estimates used in the study of the free boundary problem. In Subsection 5.3, we give an insight into the degenerate parabolic structure of $K$ by showing that the Kalman condition (3.5) is equivalent to the Hörmander condition [16]. Hereafter we refer to the simplest non-trivial example of Kolmogorov operator, i.e.

$$
\partial_{x_{1} x_{1}}+x_{1} \partial_{x_{2}}+\partial_{t}, \quad\left(t, x_{1}, x_{2}\right) \in \mathbb{R}^{3},
$$

as the prototype for the general class. Operator in (5.2) is of particular interest since it arises in the valuation of geometric Asian options (cf. (2.10)). 


\subsection{Group and metric structure}

We first remark that $K$ in (5.1) is invariant w.r.t. the law

$$
\zeta \circ z:=\left(t+s, x+e^{t B} y\right), \quad z=(t, x), \zeta=(s, y) \in \mathbb{R}^{N+1} .
$$

More precisely, defining the left translation operator $\ell$ by

$$
\ell_{\zeta} u(z)=u(\zeta \circ z)
$$

it is easily verified that

$$
K\left(\ell_{\zeta} u\right)=\ell_{\zeta}(K u)
$$

for any $\zeta \in \mathbb{R}^{N+1}$. Correspondingly, the fundamental solution of $K$, whose explicit expression in given in (3.6), has the following invariance property:

$$
G(\zeta ; z)=G\left(0 ; \zeta^{-1} \circ z\right)
$$

where $\zeta^{-1}=\left(-s,-e^{-s B} y\right)$ is the inverse of $\zeta=(s, y)$ in the law "o". It is clear that "o" reduces to the standard sum in $\mathbb{R}^{N}$ when $K$ is the heat operator and $B=0$. On the other hand, for the operator in (5.2), using the fact that

$$
B=\left(\begin{array}{cc}
0 & 0 \\
1 & 0
\end{array}\right)
$$

is a nilpotent matrix, we simply have

$$
\left(s, y_{1}, y_{2}\right) \circ\left(t, x_{1}, x_{2}\right)=\left(t+s, x_{1}+y_{1}, x_{2}+y_{2}+t y_{1}\right) .
$$

Next we introduce a "parabolic" norm in $\mathbb{R}^{N+1}$. Using the notations of Section 3, for $k=0, \ldots, N$ we denote by $V_{k}$ the vector space spanned by the columns of the matrix

$$
\left[\sigma_{0}, B \sigma_{0}, B^{2} \sigma_{0}, \ldots, B^{k} \sigma_{0}\right]
$$

and for $k=1, \ldots, N$ we define the subspace $W_{k}$ of $\mathbb{R}^{N}$ by

$$
V_{k}=V_{k-1} \oplus W_{k} .
$$

By the Kalman condition, there exists $m \leq N$ such that $V_{m}=\mathbb{R}^{N}$ : therefore $\mathbb{R}^{N}$ has an obvious direct sum decomposition and for $x \in \mathbb{R}^{N}$ we have

$$
x=x^{(0)}+x^{(1)}+\cdots+x^{(m)}
$$

where $x^{(0)} \in V_{0}$ and $x^{(k)} \in W_{k}$ for $k=1, \ldots, m$ are uniquely determined.

Definition 5.1. The B-norm of $(t, x) \in \mathbb{R}^{N+1}$ is defined as

$$
\|(t, x)\|_{B}=|t|^{\frac{1}{2}}+\sum_{k=0}^{m}\left|x^{(k)}\right|^{\frac{1}{2 k+1}} .
$$


For example, if $K$ is the heat equation, then (5.4) defines the usual parabolic norm

$$
\|(t, x)\|_{B}=|t|^{\frac{1}{2}}+|x| .
$$

This definition is in accord with the practical rule for the heat equation that "two $x$-derivatives are equivalent to one $t$-derivative". Note also that the heat equation is homogeneous of degree two w.r.t. the dilations in $\mathbb{R}^{N+1}$ defined as

$$
\delta_{\lambda}(t, x)=\left(\lambda^{2} t, \lambda x\right), \quad \lambda \in \mathbb{R},
$$

and the norm in (5.5) is $\delta_{\lambda}$-homogeneous of degree one.

Analogously, for operator (5.2) we have

$$
\left\|\left(t, x_{1}, x_{2}\right)\right\|_{B}=|t|^{\frac{1}{2}}+\left|x_{1}\right|+\left|x_{2}\right|^{\frac{1}{3}}
$$

so that in this case the practical rule reads " $\partial_{t}$ and $\partial_{x_{2}}$ respectively correspond to second and third order derivatives". Moreover operator (5.2) is homogeneous of degree two w.r.t. the dilations in $\mathbb{R}^{3}$ defined as

$$
\delta_{\lambda}\left(t, x_{1}, x_{2}\right)=\left(\lambda^{2} t, \lambda x_{1}, \lambda^{3} x_{2}\right), \quad \lambda \in \mathbb{R},
$$

and (5.6) defines a $\delta_{\lambda}$-homogeneous norm.

In general, the natural number

$$
Q=\operatorname{dim}\left(V_{0}\right)+\sum_{k=1}^{m}(2 k+1) \operatorname{dim}\left(W_{k}\right)
$$

is usually called the homogeneous dimension of $\mathbb{R}^{N}$ induced by $K$ : clearly, $Q=N$ when $K$ is a parabolic operator, while $N=2$ and $Q=4$ for the operator in (5.2).

\subsection{Sobolev and Hölder spaces}

We introduce some functional spaces modeled on the group and metric structure previously defined. Given a bounded domain $D$ in $\mathbb{R}^{N+1}$ and $p \geq 1$, we define the Sobolev space

$$
\mathcal{S}^{p}(D)=\left\{u \in L^{p}(D): \partial_{x_{i}} u, \partial_{x_{i} x_{j}} u, Y u \in L^{p}(D), i, j=1, \ldots, d\right\}
$$

equipped with the norm

$$
\|u\|_{\mathcal{S}^{p}(D)}=\|u\|_{L^{p}(D)}+\sum_{i=1}^{d}\left\|\partial_{x_{i}} u\right\|_{L^{p}(D)}+\sum_{i, j=1}^{d}\left\|\partial_{x_{i} x_{j}} u\right\|_{L^{p}(D)}+\|Y u\|_{L^{p}(D)} .
$$

Moreover, for $\alpha \in] 0,1\left[\right.$, we denote respectively by $C_{B}^{\alpha}(D), C_{B}^{1, \alpha}(D)$ e $C_{B}^{2, \alpha}(D)$ the spaces of $B$-Hölder continuous functions defined by the norms:

$$
\begin{aligned}
\|u\|_{C_{B}^{\alpha}(D)} & =\sup _{D}|u|+\sup _{\substack{z, \zeta, \in D \\
z \neq \zeta}} \frac{|u(z)-u(\zeta)|}{\left\|\zeta^{-1} \circ z\right\|_{B}^{\alpha}} \\
\|u\|_{C_{B}^{1, \alpha}(D)} & =\|u\|_{C_{B}^{\alpha}(D)}+\sum_{i=1}^{d}\left\|\partial_{x_{i}} u\right\|_{C_{B}^{\alpha}(D)}, \\
\|u\|_{C_{B}^{2, \alpha}(D)} & =\|u\|_{C_{B}^{1, \alpha}(D)}+\sum_{i, j=1}^{d}\left\|\partial_{x_{i} x_{j}} u\right\|_{C_{B}^{\alpha}(D)}+\|Y u\|_{C_{B}^{\alpha}(D)} .
\end{aligned}
$$


Remark 5.2. Since locally we have

$$
\frac{1}{c}|z-\zeta| \leq\left\|\zeta^{-1} \circ z\right\| \leq c|z-\zeta|^{\frac{1}{2 m+1}},
$$

the following inclusion relations among spaces of Hölder continuous functions (in the usual sense) hold:

$$
C^{\alpha}(D) \subseteq C_{B}^{\alpha}(D) \subseteq C^{\frac{\alpha}{2 m+1}}(D) .
$$

Several classical results from functional analysis have been extended to this non-Euclidean setting in 17, 25, 10. Here we state some fundamental embedding and a priori estimates for the variable coefficients operator $L$ in (3.11). These results provide basic tools for the study of the free boundary problem in Section 4 . In the following inequalities, $Q$ denotes the homogeneous dimension in (5.7), $D_{0}$ is a domain contained, with its closure, in $D$ and $c$ is a constant only dependent on $L, D, D_{0}$ and $p$.

- Interior Sobolev estimates:

$$
\|u\|_{\mathcal{S}^{p}\left(D_{0}\right)} \leq c\left(\|u\|_{L^{p}(D)}+\|L u\|_{L^{p}(D)}\right) .
$$

- Embedding theorem:

$$
\|u\|_{C_{B}^{1, \alpha}\left(D_{0}\right)} \leq c\|u\|_{\mathcal{S}^{p}(D)}, \quad \alpha=1-\frac{Q+2}{p}, p>Q+2 .
$$

\subsection{Kalman and Hörmander conditions}

We show that the Kalman condition (3.5) is equivalent to the Hörmander condition which is a well-known non-degeneracy criterion in PDEs' theory. We first introduce some terminology. We agree to identify any first order differential operator $Z$ in $\mathbb{R}^{N}$, of the form

$$
Z f(x)=\sum_{k=1}^{N} \alpha_{k}(x) \partial_{x_{k}} f(x),
$$

with the vector field $\left(\alpha_{1}, \ldots, \alpha_{N}\right)$ of its coefficients. The commutator of $Z$ with

$$
U=\sum_{k=1}^{N} \beta_{k} \partial_{x_{k}}
$$

is defined as

$$
[Z, U]=Z U-U Z=\sum_{k=1}^{N}\left(Z \beta_{k}-U \alpha_{k}\right) \partial_{x_{k}} .
$$

Hörmander's Theorem is a very general result which, in the particular case of operator (5.1), states that $K$ has a smooth fundamental solution if and only if the vector space spanned by the differential operators (vectors fields)

$$
\partial_{x_{1}}, \ldots, \partial_{x_{d}} \quad \text { and } \quad \tilde{Y}:=\langle B x, \nabla\rangle
$$


together with their commutators of any order, at any point $x$, is equal to $\mathbb{R}^{N}$. This is the so-called Hörmander condition.

For example, for (5.2) we simply have $\widetilde{Y}=x_{1} \partial_{x_{2}}$. Then

$$
\partial_{x_{1}} \sim(1,0) \quad \text { and } \quad\left[\partial_{x_{1}}, \tilde{Y}\right]=\partial_{x_{2}} \sim(0,1)
$$

span $\mathbb{R}^{2}$.

The equivalence of Kalman and Hörmander conditions is readily verified once we note that:

i) for $i=1, \ldots, d,\left[\partial_{x_{i}}, \widetilde{Y}\right]=\sum_{k=1}^{N} b_{k i} \partial_{x_{k}}$ is the $i$-th column of the matrix $B$. Moreover $\left[\left[\partial_{x_{i}}, \tilde{Y}\right], \tilde{Y}\right]$ is the $i$-th column of the matrix $B^{2}$ and an analogous representation of the higher order commutators is valid;

ii) for $k=1, \ldots, N, B^{k} \sigma_{0}$ appearing in (3.5) is the $N \times d$ matrix whose columns are the first $d$ columns of $B^{k}$.

\section{References}

[1] G. BARLES, Convergence of numerical schemes for degenerate parabolic equations arising in finance theory, in Numerical methods in finance, Cambridge Univ. Press, Cambridge, 1997, pp. 1-21.

[2] J. Barraquand and T. Pudet, Pricing of American path-dependent contingent claims, Math. Finance, 6 (1996), pp. 17-51.

[3] E. Barucci, S. Polidoro, And V. Vespri, Some results on partial differential equations and Asian options, Math. Models Methods Appl. Sci., 11 (2001), pp. 475-497.

[4] H. Ben-Ameur, M. Breton, And P. L'Ecuyer, A dynamic programming procedure for pricing American-style Asian options, Manag. Sci., (2002), pp. 48(5), 625-643.

[5] A. Bensoussan, On the theory of option pricing, Acta Appl. Math., 2 (1984), pp. 139-158.

[6] A. Bensoussan and J.-L. Lions, Applications of variational inequalities in stochastic control, vol. 12 of Studies in Mathematics and its Applications, North-Holland Publishing Co., Amsterdam, 1982. Translated from the French.

[7] M. Bramanti, M. C. Cerutti, and M. Manfredini, $L^{p}$ estimates for some ultraparabolic operators with discontinuous coefficients, J. Math. Anal. Appl., 200 (1996), pp. 332-354.

[8] M. Dai And Y. K. Kwok, Characterization of optimal stopping regions of American Asian and lookback options, Mathematical Finance, 16 (2006).

[9] M. Di Francesco And A. Pascucci, On a class of degenerate parabolic equations of Kolmogorov type, AMRX Appl. Math. Res. Express, (2005), pp. 77-116. 
[10] M. Di Francesco, A. Pascucci, And S. Polidoro, The obstacle problem for a class of hypoelliptic ultraparabolic equations, preprint AMS Acta (available on-line at http://www.dm.unibo.it/matecofin), (2007).

[11] P. Foschi And A. Pascucci, Path dependent volatility, preprint AMS Acta (available on-line at http://www.dm.unibo.it/matecofin), (2007).

[12] A. Friedman, Variational principles and free-boundary problems, Pure and Applied Mathematics, John Wiley \& Sons Inc., New York, 1982. A Wiley-Interscience Publication.

[13] M. C. Fu AND R. Wu, Optimal exercise policies and simulation-based valuation for American-Asian options, Oper. Res., (2003), pp. 51(1), 52-66.

[14] A. T. Hansen And P. L. Jorgensen, Analytical valuation of American-style Asian options, , Manag. Sci., 46(8) (2000), p. 11161136.

[15] D. G. Hobson And L. C. G. Rogers, Complete models with stochastic volatility, Math. Finance, 8 (1998), pp. 27-48.

[16] L. Hörmander, Hypoelliptic second order differential equations, Acta Math., 119 (1967), pp. $147-171$.

[17] K. Huang and R. Thulasiram, Parallel algorithm for pricing American Asian options with multi-dimensional assets, High Performance Computing Systems and Applications, 2005. HPCS 2005. 19th International Symposium on, (2005).

[18] J. E. Ingersoll, Theory of Financial Decision Making, Blackwell, Oxford, 1987.

[19] P. Jaillet, D. Lamberton, and B. Lapeyre, Variational inequalities and the pricing of American options, Acta Appl. Math., 21 (1990), pp. 263-289.

[20] L. JiAng And M. DAI, Convergence of binomial tree methods for European/American path-dependent options., SIAM J. Numer. Anal., 42 (2004), pp. 1094-1109.

[21] I. Karatzas, On the pricing of American options, Appl. Math. Optim., 17 (1988), pp. 3760.

[22] I. Karatzas and S. E. Shreve, Brownian motion and stochastic calculus, vol. 113 of Graduate Texts in Mathematics, Springer-Verlag, New York, second ed., 1991.

[23] D. Kinderlehrer and G. Stampacchia, An introduction to variational inequalities and their applications, vol. 31 of Classics in Applied Mathematics, Society for Industrial and Applied Mathematics (SIAM), Philadelphia, PA, 2000. Reprint of the 1980 original.

[24] E. Lanconelli and S. Polidoro, On a class of hypoelliptic evolution operators, Rend. Sem. Mat. Univ. Politec. Torino, 52 (1994), pp. 29-63.

[25] M. Manfredini And S. Polidoro, Interior regularity for weak solutions of ultraparabolic equations in divergence form with discontinuous coefficients, Boll. Unione Mat. Ital. Sez. B Artic. Ric. Mat. (8), 1 (1998), pp. 651-675. 
[26] M. D. MarcozzI, On the valuation of Asian options by variational methods., SIAM J. Sci. Comput., 24 (2003), pp. 1124-1140.

[27] G. H. Meyer, On pricing American and Asian options with PDE methods, Acta Math. Univ. Comenian. (N.S.), 70 (2000), pp. 153-165.

[28] K. Parrott and N. Clarke, A parallel solution of early exercise Asian options with stochastic volatility, Proceedings of the Eleventh Domain Decomposition Conference. Greenwich, (1998).

[29] A. PASCuCCI, Stochastic calculus for finance, Springer, forthcoming 2007.

[30] L. Rogers And Z. Shi, The value of an Asian option., J. Appl. Probab., 32 (1995), pp. $1077-1088$.

[31] D. W. Stroock and S. Varadhan, Diffusion processes. I: Existence and uniqueness., Commun. Pure Appl. Math., 22 (1969), pp. 345-400.

[32] P. van Moerbeke, On optimal stopping and free boundary problems, Arch. Rational Mech. Anal., 60 (1975/76), pp. 101-148.

[33] L. Wu, Y. K. Kwok, And H. Yu, Asian options with the American early exercise feature, Int. J. Theor. Appl. Finance, 2 (1999), pp. 101-111. 